\title{
Risas, brincos y volteretas: la enseñanza del circo en la escuela como actividad extracurricular \\ Laughter, jumps and pirouettes: Teaching circus at school as an extracurricular activity \\ *Caroline Capellato M elo, *Marco Antonio Coelho Bortoleto, **Teresa O ntañón Barragán \\ *U niversidade Estadual de Campinas (Brasil), **U niversidade Estadual de M inas Gerais (Brasil)
}

\begin{abstract}
Resumen: Este estudio tiene como objetivo comprender como transcurre la enseñanza del circo en el contexto educativo como actividad extracurricular, por medio de una investigación de experiencias escolares. M etodológicamente, lainvestigación se caracteriza como un estudio de casos múltiples, de naturaleza cualitativa, que contó con la observación de clases en dos escuelas privadas con registro en un diario de campo; entrevista a cinco sujetos, profesores y coordinadores/ directores de las dos escuelas; análisis documental de las planificaciones educativas. Los resultados muestran como la enseñanza del circo en el contexto extracurricular es un campo en pleno desarrollo, se presenta como una práctica con alta participación entre los alumnos, entretanto, centradaen al gunas modalidadescircenses, aér eosy manipulaciones, debido a unaformación no sistemática de los profesores responsables. La enseñanza es realizada con docentes de ambos dos sexos y se mostró como una práctica lúdica, principalmenteen los años iniciales, incluyendo presentaciones artísticas en ambas escuelas reconociendo ladimensión expresiva de dicha práctica. En ambos casos, pudimos apreciar que el circo presenta un relevante potencial educativo como posibilidad en la enseñanza extracurricular, no obstante, pensamos que todavía es necesario prestar atención ala demanda de formación específica de profesores y en la búsqueda de metodologías de enseñanza adecuadas a cada situación educativa.
\end{abstract} Palabras clave: Actividades circenses; Actividades extracurriculares; Educación Física; Educación; Expresión corporal.

\begin{abstract}
Thisstudy aims to under stand how circusteaching happens in the educational context as an extracurricular activity, through the investigation of schools experiences. Methodologically, the research is characterized as a multiple case study, qual itative in nature that included the observation of classes in two privateschools with a record in afield diary; interview with five subjects, teachers and coordinators/ directors of the two schools; documentary analysis of educational planning. The results show how circus teaching in the extracurricular context is a field in full development. It is present as a practice with high participation among the students, meanwhile, focused on some circus modal ities, aerial and manipulation, due to a nonsystematic qualification of the responsible teachers. Teaching is carried out with male and femal e teachers and was shown as a playful practice, mainly in the initial years, including artistic presentations in both schools, recognizing the expressive dimension of said practice. We note in both cases that the circus has a high educational potential as a possibility in extracurricular teaching, even so, we think that it is still necessary to pay attention to the demand for specific teacher training and in the search for teaching methodologies appropriate to each educational situation.
\end{abstract}

Keywords: Circus activities; Extracurricular activities; Physical Education; Education; Body La.

\section{Introducción}

Las actividades circenses se muestran, cada vez más, como una práctica con gran capacidad de adaptación al ambiente escolar, destacándose como un conocimiento innovador y bien recibido entre los estudiantes (Agua do \& Fernández, 1992; Kriellars, Cairney, Bortoleto, Kiez, Dudley \& Aubertin, 2019; M eller, De Araújo \& Silveira, 2015). El potencial creativo y artístico que esta práctica posee de acuerdo con diversos estudiosos como Invernó (2004), pone de manifiesto al gunas de las contribuciones de las actividades circenses cuando desarro-

Fecha recepción: 02-12-20. Fecha de aceptación: 22-02-21

Caroline Capellato Melo

carolcmelo9@ hotmail.com
Iladas en las escuelas, ya sea en asignaturas del currículo o como actividades extracurriculares ( 0 ntañón, Duprat $\&$ Bortoleto, 2012).

Este movimiento educativo que acabamos de indicar se observa en Brasil, país en el que se desarrolla este estudio, y también en muchos otros países, como en Argentina (Gómez, 2007), Alemania (Busse, 1991), Canadá(K riellars et al, 2019), Chile (Retamal, Cáceres, M orales \& M uñoz, 2012), China (Li, 2010), Dinamarca (Krabbe, 1988, España (Aguado \& Fernandes, 1992; Brozas-Polo, 1999; Invernó, 2003; 0 ntañón \& Bortoleto, 2014), Francia (Aubertin \& Funk, 2018; Fouchet, 2006; Hotier, 2003).

Tras más de una década de seguimiento de numerosas experiencias pedagógicas, notamos que la mayoría 
de los casos estudiados en estas producciones demuestran que la presencia del circo en la escuela ocurre en gran medida como contenido de la asignatura de Educa ción Física, es decir, en el contexto curricular (Caramês, 2014; 0 ntañón, Bortoleto \& Silva, 2013). Aun así, nota mos también, que, cada vez más, las actividades circenses protagonizan el ámbito extracurricular, destacándose como un importante espacio de la enseñanza del circo y, consecuentemente, como unavaliosa «puerta de entrada» para el tratamiento de estos saberes en el ambiente escolar (M unhões, Tiæen, Rizzo \& Zaim-DeMello, 2019; Takamori, Bortoleto, Liporoni, Palmen \& Cavallotti, 2010).

Como consecuencia del aumento de interés por parte de los docentes, en los últimos años, diversos estudios han propuesto sistematizaciones didácticometodológicas sobre la enseñanza de las actividades circenses en espacios escolares (Duprat, 0 ntañón \& Bortoleto, 2014; Invernó, 2004). La mayoría de estas producciones analizan experiencias docentes, describiendo, principalmente, las estrategias didácticas utilizadas en la enseñanza de estas actividades (Bortoleto, Pinheiro \& Prodócimo, 2011; Cardani, O ntañón, Rodrigues \& Bortoleto, 2017; Chioda, 2018; 0 ntañón, 2016; Santos Rodrigues, Prodócimo \& 0 ntañón, 2016).

Aun así, pocas las investigaciones que se han llevado a cabo sobre esta temática en el ámbito extracurricular, indicando que existe un vacío en la producción de conocimiento sobre este tema. De esta forma y asumiendo que las actividades circenses están siendo contempladas también en este espacio escolar, esta investigación tuvo por objetivo analizar las experiencias pedagógicas de la enseñanza de las actividades circenses en el contexto extracurricular en dos escuelas privadas de enseñanza básica en Brasil

\section{Material y método}

Este estudio fue construido a partir de una perspectiva cualitativa (Godoy, 1995), más específicamente como un estudio de casos múltiples, ya que analizamos más de un caso, con características semejantes, buscando una mayor profundidad en el análisis de cada caso (Thomas, 2011), aportando importantes influencias de sus diferencias y semejanzas (Gustafsson, 2017). El desarrollo del trabajo fue previamente aprobado por el Comité de Ética en Investigación de la U niversidad de Campinas, sobre registro CAAE: 66614417.3.0000.54041.

Considerando el locus del estudio, la escuela, se uti- lizaron los siguientes criterios de inclusión para la participación en la investigación: a) unidades escolares de diferentes regiones de Brasil (criterio geográfico), buscando la posibilidad de observar contextos diferentes sin la intención de utilizar un carácter comparativo, destacando elementos contextuales y los principios pedagógicos de cada experiencia; b) la actuación regular de los profesores con un mínimo de dos años de docencia en la unidad escolar, con la enseñanza de las activida des circenses en el contexto extracurricular (criterio especificidad).

De entre las treinta y cinco instituciones escolares encontradas en la fase inicial del estudio, se selecciona ron dos: Escuela 1, localizada en el estado de São Paulo, Brasil (región Sureste) en la que observamos dieciocho clases de una hora cada una, en seis días diferentes, en la que participan en media 250 alumnos y la Escuela 2 localizada en el estado de Paraná, Brasil (región Sur), en la que observamos diez clases de una hora cada una, durante dos días', en laque participan en media 50 alumnos.

Las observaciones directas de las actividades fueron registradas en un diario de campo que, posteriormente, fue organizado de manera que permitiese un análisis con intersección de las informaciones, entre el cotidia no de las clases y las informaciones de las entrevistas (Minayo, 1992). Por otro lado, las entrevistas semiestructuradas con los profesores y coordinadores/ directores de las instituciones, que en total fueron cinco, fueron grababas en audio, transcritas y analizadas posteriormente con el empleo de la técnica de análisis categorial de contenido de acuerdo con losprocedimientos descritos por Bardin (2011) y K rippendorff (2004). Las entrevistas realizadas con los docentes contenían preguntas sobre la formación de los profesores, sus estrategias pedagógicas durante las clases, contenidos y modalidades que enseñaban, entre otras. Ya las entrevistas dirigidas a los coordinadores se centraban en la rela ción gestión/ profesor y en la visión que la escuela tenía sobre las actividades circenses realizadas en los centros.

Para el análisis de los documentos, que incluyó los proyectos pedagógicos de las escuelas, las planificaciones educativas de los profesores y folders de divulga ción de los proyectos y sus presentaciones, fue utilizada la misma técnica que en las entrevistas.

Cabe indicar que, tras la fase preliminar de lectura y sistematización de los dados, obtuvimos el siguiente sistema de categorías: a) diversidad de modalidades circenses enseñadas; b) equipamientos empleados y espacios disponibles; c) estrategias pedagógicas; d) uso de 
juegos y actividades lúdicas; e) presentación artística como síntesis del proceso.

\section{Análisis y discusión}

\section{Caracterización de las escuelas}

La Escuela 1 es una institución privada fundada en 2005 que posee un amplio espacio físico (aproximada mente, $18.000 \mathrm{~m}^{2}$ ). Con un carácter innovador, la institución se presenta con muchas aulas de paredes retráctiles, pizarras digitales y un altavoz en el que suenan canciones que homenajean a un artista diferente cada mes. Una característica muy importante en esta escuela son las actividades extracurriculares, que son denominadas por el centro como «actividades complementares». Según comentó la coordinadora, tienen este nombre porque «no es extra, sino que complementa y amplia aquello que creemos no curricular» (extraído de la entrevista con la coordinadora, líneas 30-31). Las actividades ofrecidas son: capoeira, escala da, judo, fútbol, guitarra, ballet, parkour, culinaria, yoga, patinaje, teatro, deportes de aventura y, de modo destacado, el circo.

Las actividades circenses poseen un papel prominente entre las actividades complementares ya que, además de existir desde que la institución fue fundada, es la actividad que congrega más de doscientos alumnos inscritos regularmente, lo que representa una cuarta parte de los estudiantes de la escuela. Notamos que el apoyo institucional y la sintonía entre los profesores y la coordinación permiten que el proyecto esté siempre evolucionando.

O bservamos la existencia de un espacio dedicado exclusivamente a ella, que actual mente es una carpa de circo y posee una gran variedad de materiales, contemplando las diferentes modalidades circenses como telas, lira, trapecio, cuerda lisa, cama elástica, mini trampolín, bola de equilibrio, zancos, rola-bola, pañuelos, bolas, mazas, aros, devilstick, diábolo, platos de equilibrio y colchones de diversos grosores.

El responsable de las actividades circenses en la escuela es un profesor que trabajó en un circo durante cinco años y se formó en Educación Física en una universidad privada, en la que pudo especializarse en la pedagogía circense por medio de un proyecto que funcionaba en lainstitución. El profesor coordinaun equipo de otros cuatro profesores (dos hombres y dos mujeres), juntos, dan clases de circo en laescuela desde 2013, para todos los cursos escolares (de primaria a bachillerato). Todos los profesores son contratados de manera tercerizada por la escuela, o sea, con un régimen distinto a los docentes que actúan en la enseñanza curricular.

Durante la observación de las clases acompañamos doce grupos que tenían un mínimo de ocho alumnos y un máximo de treinta y tres por grupo. La separación de las clases sucedía de la siguiente forma: Grupo de «Experimentación»: con alumnos de Primaria, en el que la clase era organizada en circuitos o estaciones y los alumnos eran divididos de acuerdo con el número de profesores, de modo que cada profesor permanecía en una modalidad (estación) y, durante la clase, todos losgrupos circulaban por todas las estaciones. Y el grupo de «Perfeccionamiento»: con alumnos de Secunda ria y Bachillerato, en el que los alumnos elegían una modalidad circense en la que querían especializarse, pudiendo permanecer en esta durante toda la clase.

Por otro lado, la Escuela 2 es una escuela privada, fundada en 1953 y ocupa un espacio de $14.000 \mathrm{~m}^{2}$. Las actividades extracurriculares de esta institución también poseen un papel relevante en la escuela, son: fútbol sala, vóleibol, balonmano, baloncesto, teatro, coral, capoeira, judo, música, baile y circo.

También contratados de forma tercerizada, los responsables de las actividades circenses en la institución son dos profesores, ambos artistas profesionales y formados en Educación Física en universidades privadas. El conocimiento pedagógico de las actividades circenses se adquirió por medio de talleres en convenciones de circo y malabares, ya que en la universidad no tuvieron acceso a este conocimiento.

Vale la pena destacar que, actualmente, en la región sur de Brasil, donde está situada esta escuela, encontramos algunas posibilidades de formación continuada, en escuelas de circo, proyectos sociales (Duprat, 2014) y, también cursos de posgrado, como los ofrecidos por dos universidades de la región. De modo general, esas posibilidades formativas refuerzan la idea que la práctica del circo viene ganando importancia en las últimas décadas (Tucunduva \& Bortoleto, 2019) y por otro lado, la gran necesidad de formación del profesorado en esta área, que hasta hace poco tiempo, no había sido muy explorada.

Los profesores, un hombre y una mujer, ofrecen las clases en dicha escuela desde 2014 para alumnos de primaria a bachillerato. De los aproximadamente mil seiscientos alumnos que la institución atiende, cerca de sesenta al umnos realizan las clases de actividades circenses. Los profesores comentaron que esta fue laúnica institución de la ciudad que abrió las puertas para desarrollar el proyecto. A diferencia de la anterior, vemos que ha 
sido una propuesta presentada por los docentes.

Los profesores eran los responsables de aportar su propio material para las clases, ya que no había equipamiento específico en la escuela y tampoco hubo interés en comprarlo alo lardo de esos años. De hecho, ese fue un problema apuntado por los profesores. Entretanto, observamos la enseñanza de diferentes modalidades circenses: telas acrobáticas, trapecios, lira y colchones, aros, mazas, pelotas, diábolos, devil stick, plato chino, rola-bola, monociclo y jirafa (monociclo alto). Además diversos atrezos estaban disponibles para ser usados en los procesos creativos.

N ormal mente, el espacio donde se desarrollaban las clases era un pabellón cubierto, que estaba reservado para estas actividades los jueves y viernes. Si por cualquier motivo el espacio se utilizaba para otras finalida des, los profesores podían utilizar la sala de danza y música, que, por ser un lugar más pequeño y con poca altura, requería otras formas de uso, aunque también permitía otras actividades como, por ejemplo, la realización de pequeñas presentaciones, ya que este espacio contaba con un escenario.

En esta escuela, las clases se organizaban en seis grupos con un mínimo de cinco alumnos y un máximo de trece alumnos por grupo. La separación de los grupos era por edad, los grupos de Primaria realizaban las cla ses en conjunto, practicando una o dos modal idades en cada clase. Los grupos de Secundaria y Bachillerato también practicaban las actividades propuestas por los profesores, pero tenían mayor autonomía para practicar la modalidad de su preferencia, teniendo un modelo de clase más libre.

\section{Caminos que se cruzan para la enseñanza del circo}

LaEscuela 1 posee un amplio apoyo institucional en el desarrollo de las actividades circenses, siendo ese un proyecto ya consolidado en la misma. Por el contrario, la Escuela 2 depende en gran medida de los profesores responsables para que la actividad continúe siendo rea lizada. Esta característica ya fue señalada en estudios anteriores con otras escuelas brasileñas (Cardani, 2018; O ntañón \& Bortoleto, 2014; Santos Rodrigues, 2018), de hecho en la mayoría de casos estudiados, la iniciativa de enseñar las actividades circenses dependía del interés y la proactividad del profesorado. Por ese motivo, señalamos la particularidad de la Escuela 1, en el que las actividades circenses están presentes desde la fundación de la institución, teniendo su presencia e importancia consolidada, y su desarrollo independientemente de profesores 0 gestores que actúan en ella. N os fue informado que hubo diversos docentes en la Escuela 1, sin que eso representase la interrupción de la enseñanza del circo.

La forma en la que estos profesores se formaron y llegaron a las escuelas es diversa, aun así, la formación artística realizada en paralelo durante la graduación en Educación Física fue reportada por todos los docentes entrevistados. Es importante mencionar que la forma ción direccionada a la pedagogía del circo no siempre es ofrecidapor launiversidad. Sobreesto, Fernandes(2014) y Tucunduva y Bortoleto (2019) comentan que el número de profesores e instituciones de enseñanza superior que trabajan con las actividades circenses en el contexto de formación de profesores en los cursos de Educación Física en Brasil todavía es pequeño, aunque está aumentando. De hecho, los diferentes espacios de actuación del profesional deEducación Físicapasaron, como debate Duprat (2014), a demandar más y más profesores para la enseñanza de las actividades circenses, produciendo otros espacios que ofrecen formación continuada, contribuyendo en la preparación de muchos docentes (Bortoleto et al, 2020).

0 bservamos también que los profesores de la Escuela 1 no poseen planificación ni evaluación sistematizados y en la Escuela 2, nos encontramos con una planificación macro sobre las modalidades que se trabajan, que es enviada a los padres para que entiendan las actividades, siendo más una guía explicativa de lo que los alumnos van a aprender que una planificación pedagógica de los contenidos.

De esta forma, resaltamos la importancia de sistematizar y presentar en la forma de cursos específicos podría facilitar la acción de los profesores, especial mente en la organización del proceso pedagógico. En ese sentido, podemoscitar aCardani (2018), Duprat y Pérez Gallardo (2010), Invernó (2003), O ntañón (2016), Simon (2000), Vendruscolo (2009), entre otros, que presentan en sus estudios diferentes opciones para la práctica de las actividades circenses. 0 tros materialestambién træen importantes contribuciones didáctico-pedagógicas y estrategias pedagógicas para auxiliar alos profesores, como es el caso de Alonso \& Barlocco (2013) o Bortoleto, Pinheiro \& Prodócimo (2011).

Lo mismo destacamos sobre la evaluación sistemá tica de las clases. En este punto, cabe resaltar que no estamos en busca de una evaluación característica de enseñanzas tradicionales, ligadas a conceptos o clasifica ciones, sino a una evaluación de carácter más cual itativo, defendemos que sea real izada una evaluación conec- 
tada a las condiciones de enseñanza, es decir, que sirva tanto para que los al umnos optimicen sus aprendizajes como para el profesor, indicándole las decisiones acertadas o las que necesitan ser repensadas (Cardani, 2018). Aun así, es necesario destacar que, en ninguna de las escuelas fueron identificados procesos de evaluación.

Creemos que la falta de esta sistematización, planificación y evaluación, aliada con las afinidades persona les de los profesores por al gunas modalidades circenses específicas, causó también que la enseñanza se concentrase en un conjunto restricto de modal idades circenses. En la Escuela 1, en la mayoría de las clases, la cama elástica y las telas fueron las modalidades más trabajadas; ya en la Escuela 2, la manipulación de objetos y las modalidades aéreas (telas y trapecio) fueron las actividades más observadas. Ese registro se confirma, además con las palabras de la profesora: «enseñamos principalmente malabares y aéreos, porque es en lo que tenemos más dominio, aunque también trabajamos con representación en juegos de clown y equilibrios» (extraído de la entrevista con la profesora, líneas 81-83).

Entendemos que esta característica se mostró liga da a la formación y experiencia de los profesores, los cuales actúan 0 actuaron artísticamente en las modalidades que luego son las más trabajadas en las clases. Sin embargo, pensamos que estas preferencias representan cierta limitación, principalmente si consideramos la amplia variedad de posibilidades que el circo posee, como destaca Bortoleto (2017). En ese mismo estudio el autor discute que los profesores tienden a abordar pocas prácticas, normalmente, aquellas con las que tuvieron contacto previo y se siente con mayor dominio. Entendimos que, en contraposición a lo observado, es por medio de la diversidad que las actividades circenses presentan, que los al umnos podrán identificarse con una u otra modalidad, ampliando el interésy, por consiguiente, la participación activa (Kriellars et al 2019).

En relación con las estrategias didácticas utilizadas por los profesores, observamos que los contenidos más trabajados en la Escuela 1 eran enseñados para las clases de experimentación y perfeccionamiento, entretanto notamos que en el grupo de experimentación la enseñanza era realizada de manera más lúdica. Como podemos ver en el siguiente apunte del diario de campo:

Cuatro estaciones - la tela con trenza era el otoño y los al umnos se balanceaban en la posición de escuadra. Después, los alumnos pasaban con pequeños paraguas por unas maderas en el suelo que hacían de cuerda floja. La próxima estación era el invierno en la bola de equilibrio, que los al umnos giraban para el polo norte y para el polo sur y, después de esto, pasaban por aros en el suelo, que eran caracterizados como islas. Llegaban, entonces, a la primavera, en la que se exploraban los pañuelos (lanza-agarra; lanza-pal mada-agarra; lanza-dos palmadas-agarra; lanza-gira-agarra; lanza-palmada debajo de la pierna -agarra; lanza-agarra el pañuelo del compañero de al lado). La profesora iba dando los comandos y ellos iban haciéndolo. D espués, pasaban por la cama elástica e iban hasta el verano, estación en la que había dos aros, uno con el que los alumnos debían realizar un salto agrupado (aro en horizontal) y el otro, una voltereta hacia delante (aro en vertical), acabando en el trapecio, donde realizaban un columpio y hacían un aterrizaje en una «x» imaginaria delante del trapecio (extraído del diario de campo, líneas 197-223).

Por otro lado, en las clases de perfeccionamiento, la estrategia de enseñanza utilizada era la demostración, en la que los profesores realizaban el ejercicio propuesto y los alumnos lo repetían. Pensamos que, por medio de los juegos circenses, los alumnos pueden vivenciar aspectos relevantes (como técnica, estética, lógica de la práctica...) de manera lúdica, agradable y motivadora, contribuyendo al proceso de aprendizaje (Abrahão, 2011; Bortoleto, Pinheiro \& Prodócimo, 2011; Ontañón, Lopes, Santos Rodrigues, Cardani \& Bortoleto, 2019).

De cualquier forma, es necesario destacar que, en las clases de perfeccionamiento, los alumnos poseen una independencia mayor de los profesores para realizar las actividades. Aun así, pudimos observar en diversas ocasiones que los alumnos mostraban desinterés en practicar las modalidades elegidas por los profesores, o incluso aburridos, como muestra el siguiente trecho del diario de campo:

Los cinco alumnos que estaban sin direccionamiento estuvieron, la mayor parte del tiempo, en la cama elástica. Se les dijo que solo deberían usarla uno cada vez, aunque no siempre pasaba eso. También hicieron plato chino. En algunos momentos, se les observo tumbados en los colchones de las lateral es mientras los otros saltaban. Hubo un niño que cogió un rola-bola en la sala de materiales, pero, sin saber lo que hacer, lo guardó y cogió las pelotas de malabares. Las guardo de nuevo y volvió a la cama elástica (extraído del diario de campo, líneas560-565).

Esta información, choca con lo observado en las cla ses con los alumnos más pequeños que, aun teniendo muchos más alumnos por clase, siempre parecían entusiasmados y hacían todas las actividades propuestas. Asimismo, nos parece interesante resaltar que la construcción de la autonomía debe explorar la curiosidad y la 
inquietud de los alumnos(Freire, 1996), considerando a los profesores como mediadores del aprendizaje, ga rantizando, por medio da la planificación, que los alumnos consigan realizar las diversas prácticas con seguridad, proponiendo nuevos desafíos y motivaciones para la práctica del circo, como también lo sugieren Da Silva, De Souza, Telles, Krug y Kunz (2016).

Algunas similitudes fueron observadas en la Escuela 2. A menudo los profesores realizaban actividades poco convencional es con los objetos, como castillo de aros y dinosaurio con mazas y aros. De esta forma, la parte lúdica y el desafío de construir algo cada vez mayor era tan envolvente que los alumnos ni siquiera percibían que estaban mejorando en aquella modalidad. Entretanto con los alumnos de Secundaria y Bachillerato, no encontramos la misma estrategia metodológica, sino el aprendizaje por medio de la demostración, en la que los profesores proponían a los alumnos la repetición del ejercicio demostrado. Con los alumnos más mayores también observamos que tenían mayor autonomía para entrenar en la modalidad de su preferencia. Esto ocurre, por medio de la exploración libre, en la que los alumnos se ayudan mutuamente para encontrar soluciones y realizar con éxito los desafíos propuestos por el profesor, que los incentivaba a crear movimientos cada vez más complejos, metodología también defendida por Invernó (2003) y presentada en O ntañón y Bortoleto (2014).

Con relación a las estrategias pedagógicas lúdicas, pudimos observar que están más dirigidas a los alumnos de la Enseñanza Fundamental I y en ciertas modalidades más que en otras. Varios autores defienden la importancia del juego y de estrategias pedagógicas lúdicas en la enseñanza de las actividadescircenses. (D uprat, 2007; Bortoleto, Pinheiro \& Prodócimo, 2011; Santos Rodrigues, Prodócimo \& 0 ntañón, 2016).

En este punto, cabe matizar que compartimos las ideas expresadas por Emerique (2004) y Grillo, Spolaor y Prodócimo (2016) cuando entienden que lo lúdico alcanza peculiaridades que tal vez otras estrategias no consiguen al canzar con tanta efectividad, tales como: el placer funcional, el desafío y la libertad de expresión.

Reflexionando sobre el éxito del uso de situaciones lúdicas con los más pequeños, nos preguntamos sobre la ausencia de este tipo de metodología con los alumnos de secundaria y bachillerato. En estudios sobre el juego, Grillo (2018) comenta como el juego y lo lúdico toda vía se tiene como una dimensión puramente infantil (infantilización del juego), tanto es así, que el juego no está presente en la mayor parte de los documentos de
Educación Física de Bachillerato (cuando aparece, es tenido como utilitario). De igual manera, en las actividades extracurriculares, que no poseen documento específico que las orienten, los profesores estudiados optaban por no utilizar el juego como una forma de enseñar las diversas actividades circenses, a pesar de que varios de ellos son graduados en Educación Física y conocen la importancia del mismo.

En lo que se refiere a la centralidad del juego en modalidades específicas como, por ejemplo, las modalidades aéreas, no fue observado el uso de esta estrategia durante las clases, que se basaba en la enseñanza enfoca da en la repetición de figuras y habilidades. Resaltamos que los juegos aéreos ya han sido explorados por varios autores, como comenta Bortoleto (2008), y se muestran como una alternativa interesante a los ejercicios de repetición, predominantes en la enseñanza de estas modalidades. Defendemos un tipo de educación activa y transformadora, ya sea en el ámbito escolar, como en el ámbito extraescolar, de esta forma, destacamos que es necesaria la formación crítica y reflexiva de los profesores, de manera continuada, permitiendo la reflexión sobre la actividad docente y revisando, por ejemplo, la posibilidad de incluir los juegos en diferentes situaciones, así como de otras metodologías o recursos de enseñanza, siempre buscando ofrecer a los niños y jóvenes la posibilidad de ser sujetos activosy autónomosen el proceso de aprendizaje.

0 tro punto que nos llamó la atención en las escuelas fue la importancia y relevancia dada a los espectáculos de circo que se realizaban en las clases. En la escuela 1, la institución contrataba un equipo con diseñador de vestuario, director artístico y profesionales de ilumina ción y grabación. Durante conversaciones con el profesor, nos contó que los alumnos tenían autonomía para la creación de los números que iban presentar en el espectáculo. No obstante, nos llamó la atención que ese proceso creativo no fue observado como parte de la propuesta pedagógica desarrollada durante el período que estuvimos acompañando las clases.

Ya en la escuela 2, la presentación de final de curso también fue destacada como un elemento importante para el desarrollo de la actividad circense en la escuela. Como nos contó la profesora, se trata de un producto que permite mostrar a la comunidad lo que los alumnos aprendieron durante todo el año.

Además de la presentación, los alumnos también realizaron otras acciones durante el año de 2019. Los profesores comentaron sobre una presentación en el teatro de la ciudad, abierta al público, en la que los 
alumnos también participaron en algunas intervenciones, y, finalmente, la participación en el festival de circo social en la ciudad deToledo (Brasil), situada también en el Estado de Paraná. Los profesores comentaron que las presentaciones son montadas a partir de las activida des realizadas durante todo el año con clases de expresión corporal: «comienza en la clase de payaso, hacemos juegos de payaso y, de esos juegos salen mini-teatros» (extraído de la entrevista con el profesor, líneas 240241).

Compartimos la idea de que la práctica de las presentaciones es interesante, principalmente si pensamos en el desarrollo de la expresión corporal, o en aprender a exteriorizar sensaciones y emociones, como también defiende Santos Rodrigues, M artinsy DeM arco (2020). Melo et al (2019) sostienen que, en este proceso pedagógico, es fundamental que los al umnos participen activamente en el proceso de creación de la presentación, siendo riquísima la posibilidad de participar en le elección de las modalidades, de los materiales, del tema, de la música, etc. Esta misma perspectiva, se encuentra también en la propuesta elaborada por el Gobierno de Uruguay recientemente (Alonso \& Barlocco, 2013). Parece, entonces, que, es por medio de la interacción entre los alumnos durante el proceso de construcción de los espectáculos, como se trabaja, al mismo tiempo, la solidaridad, el respeto a los demás y la tolerancia hacia los compañeros.

Somos conscientes también, de la importancia que estas presentaciones tienen en lo que se refiere al fomento de lacreatividad de losalumnos (O ntañón, 2016), contribuyendo para una educación artística, estética y que participa en el desarrollo de la expresión corporal (Bortoleto, 2011). Entre tanto, notamos que, además de estos objetivos, las actividades circenses en el contexto extracurricular ocupan un espacio escolar en el que los familiares de los alumnos que participan en estos proyectos quieren (y hasta exigen) ver un producto final, el espectáculo de fin de curso, y esta responsabilidad, recae casi que exclusivamente sobre los profesores.

Finalmente enfatizamos en como las actividades extracurriculares, están en muchos casos, convirtiéndose en un espacio utilizado para promocionar las escuelas, es decir, como una técnica de atraer nuevos alumnos, así como para fidelizar o incluso ampliar el ingreso (financiero) de la institución. En el caso de las actividades circenses esto puede provocar, por ejemplo, una sobrevaloración de los resultados obtenidos, provocando una cierta presión sobre los profesores y alumnos para la realización de un buen espectáculo y consecuentemente una menor atención en el proceso de enseñanza. En el estudio realizado por Lopes (2009) encontramos también impresiones parecidas sobre las actividades extracurriculares, aunque realizado en un ámbito deportivo, la autora enfatiza los intereses económicos y también la comodidad que las actividades extracurriculares traen para los familiares de los alumnos.

\section{Consideraciones finales}

Presentamos en esta oportunidad, los hallazgos de una investigación realizada en dos escuelas localizadas en Estados brasileños diferentes, con particularidades propias, pero también con algunas similitudes. Desta camos en la Escuela 1 el reconocimiento y apoyo de la institución para con las actividades circenses. Por otro lado, en la Escuela 2, notamos que las actividades circenses son también muy bien recibidas por la dirección de la escuela que, desde su inicio en 2014, actúa para mantenerlas activas. Aun así, las actividades solo son posibles debido al «préstamo» de diferentes materiales/ equipamientos de propiedad de los profesores, condición distinta a la obser vada en la escuela anterior.

De manera general, estos dos casos, aunque presenten diferencias y particularidades contextuales, mostraron también similitudes que pueden ser de ayuda a otros investigadores, profesores y gestores en el proceso de implementación o reflexión sobre las diferentes posibilidades de la enseñanza de las actividades circenses en el ambiente escolar.

La formación de los profesores que actúan con la enseñanza de las actividades circenses todavía es escasa y dependiente de iniciativas individuales de los docentes. Para acompañar la creciente demanda de los espacios educativos que ofrecen estas prácticas, incluyendo la escuela, no parece urgente el ofrecimiento de programas específicos.

Considerando la gran diversidad de posibilidades que las prácticas circenses presentan, es necesario pensar la formación de los profesores buscando la pluralidad de los contenidos y metodologías, de modo que se puedan construir estrategias para cada contexto educativo.

Aunque desarrollados en realidades diferentes, los casos estudiados en esta investigación muestran que ambos proyectos funcionan en instituciones privadas, confirmando ser esta una práctica que «agrada a su público» (participantes y familiares), característica que no puede ser ignorada en el ámbito escolar, sea curricular 
o extracurricular. Vemos, por lo tanto, el surgimiento de una nueva forma de «tratar» el arte circense, como parte del proceso de formación ofrecido por las escuelas, una forma relativamente nueva, que viene desarroIlándose rápidamente y que merece atención de los estudiosos del área. Por este motivo, nos parece importante que este tipo de proyectos continúen siendo estudiados, para poder entender, cada vez mejor, como las actividades circenses encuentran cada vez más oportunidades en los espacios educativos y recreativos.

Por fin, nos parece que será por medio de la experiencia producida en diferentes escuelas que podremos conocer los procesos pedagógicosque permitan sorprender y encantar a niños, jóvenes y, por qué no, adultos.

\section{Referencias}

Abrahão, S. R. (2011). Valoración de las actividades circenses en la formación del profesorado de Educación Física: Una propuesta para la transformación social en la escuela (Tesis de doctorado). Facultad de Formación del Profesado, Universidad de Barcelona, Barcelona.

Aguado, X., \& Fernández, A. (1992). U nidades didácticas para primaria II: Los nuevos juegos de siempre- El mundo de los zancos, Juegos malabares y Juegos de calle. Zaragoza, España: Inde Publicaciones.

Alonso, V. \& Barlocco, A. (2013). A portes parala crea ción de una función de circo In Encastres: propuestas para una escuela en juego - circo. Coordinación técnica MIDES-CEIP, U ruguay. Recuperado de http:/ / ww w. ceip. edu.uy/ documentos/2013/ ProgramaM aestrosComunitarios/ encastres/ circo_i mprenta3.pdf

Aubertin, P., \& Funk, A. (2018). Les arts du cirque dans le cadre de l'enseignement primaire au Canada: genèse d'une recherche et $d$ ' un projet d'innovation sociale. In FROISSANT, T., \& THOMAS, C. Arts du cirqueet spectacle vivant: les formations en arts du cirque et en activitiés physiques artistiques (1) 157-174, Reims, Francia: Épure.

Bardin, L. (2011). Análise de conteúdo. San Pablo, Brasil: Edições 70.

Bortoleto, M . A. C. (O rg.). (2008). Introdução à pedagogia dasatividadescircenses. Jundiaí, Brasil: Fontoura, 2008.

Bortoleto, M. A. C. (2011). Atividades circenses: notas sobre a pedagogia da educação corporal e estética. Cadernos de formação RBCE, 2(2), 46-55. Recuperado de http:/ / oldarchive. rbceonline.org. br/ index. php/ cadernos/ article/ view/ 1256/ 651

Bortoleto, M. A. C. (2017). Um encontro entre 0 funâmbulo e o praxiólogo: ideias para mestres e discípulos. In FERREIRA, L. A., \& RAMOS, G. N. S. Educação Física Escolar e praxiologia motriz: compreendendo as práticas corporais (22), 55-80. Curitiba, Brasil: CRV.

Bortoleto, M. A. C., Pinheiro, P. H. G. G., \& Prodócimo, E. (2011). Jogando com o circo. Várzea Paulista, Brasil: Fontoura.

Bortoleto, M.A.C., O ntañón, T. B.; Cardani, L.T.; Funk A; Melo, C.C., \& Santos Rodrigues, G. (2020). Gender participation and preference: a multiplecase study on teaching circus at PE in Brazilians schools. Front. Educ. 5:572577. doi: 10.3389/ feduc. 2020.572577.

Brozas, M. P. (1999). La recuperación del trapecio en la educación física: de la historia a la didáctica. Lecturas: EFyD, 4(17).

Busse, H. (1991). Artistik: Hohe schule der Körperkunst. Cidade, País: Zentralhaus-Publ, 1991.

Caramês, A. S. (2014). Professores na corda bamba: As atividades circenses na formação inicial enquanto conteúdo da Educação Física (Tesis de maestria). Centro de Educação Física e Desportos, U niversidade Federal de Santa Maria, Santa Maria.

Cardani, L. T. (2018). Atividades circenses: compartilhando práticas pedagógicas no ensino fundamental (Tesis de maestria). Faculdade de Educação Física, Universidade Estadual de Campinas, Campinas.

Cardani, L. T., 0 ntañón, T. B., Rodrigues, G. S., \& Bortoleto, M. A. C. (2017). Atividades circenses na escola: a prática dos professores da rede municipal de Campinas-SP. Revista Brasileira de Ciência e M ovimento, 25(4), 128-140. Recuperado de https:/ / portal revistas. ucb.br/ index. php/ RBCM / article/ view/ 7723

Chioda, R. A. (2018). U ma aventura da alegria e do risco: narrativas de um professor de Educação Física sobre o ensino das atividades circenses (Tesis de doctorado). Faculdade de Educação Física, U niversidade Estadual de Campinas, Campinas.

Da Silva, D. O., De Souza, A., Telles, C., Krug, H. N., \& Kunz, E. (2016). A tividade circense na escola: caminhos àorganização didáticaa partir da concepção crítico-emancipatória. LICERE - Revista do Programa de Pós-graduação Interdisciplinar em Estudos do Lazer, 19(1), 306-326. Recuperado de https:// repositorio. ufsm. br/ handle/ 1/ 60.

Duprat, R. M. (2007). Atividades circenses: possibilidades e perspectivas para a educação física escolar. (Tesis de maestria). Faculdade de Educação Física, 
Universidade Estadual de Campinas, Campinas.

Duprat, R. M. (2014). Realidades e particularidades da formação do profissional circense no Brasil: rumo a uma formação técnica e superior (Tesis de doctorado). Faculdade deEducação Física, U niversidadeEstadual de Campinas, Campinas.

Duprat, R. M., O ntañón, T. B., \& Bortoleto, M. A. C. (2014). A tividades Circenses. In González, F. J., Darido, S. C., \& De O liveira, A. A. B. (O rgs.). Ginástica, dança e atividades circenses (3), 119-157. Maringá, Brasil: Editora da Universidade Estadual de Maringá - EDUEM.

Duprat, R. M., \& Perez Gallardo, J. S. (2010). Artes circenses no âmbito escolar. Ijuí: Ed. U nijuí.

Emerique, P. S. (2004). Aprender a ensinar por meio do Iúdico. In SCHWARTZ, G. M. (O rg.). Dinâmica Lúdica - Novos O lhares, 3-17. Barueri, Brasil: Manole.

Fernandes, R. C. (2014). D otecido à lona: aspráticascircenses no tear da formação inicial em Educação Física (Tesis de doctorado). Faculdade de Educação, U niversidade Estadual de Campinas, Campinas.

Fouchet, A. (2006). Lasartes del circo: una aventura pedagógica. Buenos Aires, Argentina: Stadium.

Freire, P. (1996). Pedagogia da autonomia. Rio de Janeiro, Brasil: Editora Paz eTerra.

Godoy, A. S. (1995). Pesquisa qualitativa: Tipos fundamentais. Revista de administração de empresas, 35(3), 20-29. Recuperado de https:// www. scielo.br/ pdf/ ra/ v35n3/ a04v35n3.pdf

Gómez, P. (2007). El circo en la escuela como proyecto. U na propuesta significativa para el desarrollo de los contenidos delaEducación Físicaescolar. Revista Educación Física y Deportes, 115(12).

Grillo, R. D. M. (2018). Mediação semiótica ejogo na perspectiva histórico-cultural em Educação Física escolar (Tesis de doctorado). Faculdade de Educação Física, Universidade Estadual de Campinas, Campinas.

Grillo, R. D. M., Spolaor, G., \& Prodócimo, E. (2016). La cultura lúdica y la educación física escolar. Revista Ludicamente, 5(10). Recuperado de https:/ / dialnet.unirioja. es / servlet/ articulo?codigo $=5689539$.

Gustafsson, J. (2017). Single case studies vs. multiple case studies: A comparativestudy. Recuperado dehttp:/ / diva portal.org/smash/get/diva2:1064378/ FULLTEXT01.pdf

Hotier, H. (O rg). (2003). La fonction éducative du cirque. Paris, Francia: L'Harmattan.

Invernó, J. C. (2003). Circo y Educación Física: otra forma de aprender. Barcelona, España: INDE.

Invernó, J. C. (2004). El circo en laescuela. RevistaTandem - Didáctica de la Educación Física, (16), 72-82.

Krabbe, P. (1988). Hopla Gogler. O dense, Dinamarca: Fins Paedagog-Seminarium.

Kriellars, D. J., Cairney, J., Bortoleto, M. A., Kiez, T. K., Dudley, D., \& Aubertin, P. (2019). The Impact of Circus Arts Instruction in Physical Education on the Physical Literacy of Children in Grades 4 and 5. Journal of Teaching in Physical Education, 38(2), 162170. Recuperado de https:// journals. humankinetics. com/ view/ journals/ jtpe/ 38/ 2/ article-p162.xml

Krippendorff, K. (2004). Content analysis: an introduction to its methodology (2a ed.). California, EEUU: Sage Publications.

Li, C. (2010). Serving PE teachers' professional learning experiencesin social circus. N ew H orizonsin Education, 58(1), 108-119. Recuperado de https:// eric. ed. gov/ ?id=EJ 893715

Lopes, P. R. (2009). M otivação eginástica artística formativa no contexto extracurricular (Tesis de maestria). Escola de Educação Física e Esporte, U niversidade de São Paulo, San Pablo.

Marconi, M. A., \& Lakatos, E. M. (1982). Técnicas de pesquisa: Planejamento eexecução de pesqui sas, amostragens e técnicas de pesquisas, elaboração, análise e interpretação de dados. San Pablo, Brasil: Atlas.

Meller,V. A, DeAraújo, L. C. G, \& Silveira, R. E. (2015). Atividades Circenses na Educação Física escolar. Revista de Divulgação Interdisciplinar (ed. esp.) , 3(2). Recuperado de http:/ / www. saosebastiao.sp. gov. br/ ef/ pages/ I inguagem/ expressividade/ atividades_circenses/Leitura/ Atividade\%20circenses\%20na\%20Educa\%C3\%A7\%C3\%A 30\%20F\%C3\%ADsica\%20escola.\%20experi\%C3\%AAn cia\%20pedag\%C3\%B3gica\%20no\%20PIBID.pdf

Melo, C. C. (2020). Atividades circenses: compartilhando práticas pedagógicas no ensino extracurricular da escola básica. (Tesis de maestria). Faculdade de Educação Física, U niversidade Estadual de Campinas, Campinas.

Melo, C. C., Rodrigues, G. S., Cardani, L. T., Barreto, M. L., \& Bortoleto, M. A. C. (2019). Invertendo a lógica: crianças ensinando circo para adultos-todos aprendendo. Educación Física y D eporte, 38(2). Recuperado de https:/ / revistas. udea. edu. co/ index. php/ educacionfisicaydeporte/ article/ view/ 339035.

M inayo, M. C. (1992). 0 desafio do conhecimento: pesquisa qualitativa em saúde. Rio de Janeiro, Brasil: Hucitec; 
Abrasco.

Munhões, L. C. S., Tiaen, M. S., Rizzo, D.T. S. \& Zaim-

De-M ello, R. (2019). A tividades circenses no contraturno escolar: um estudo com estudantes do ensino médio. Lecturas: Educacion Física y Deportes, 24(255), 2-12. Recuperado de https:// www. efdeportes.com/ efdeportes/ index. php/ EFD eportes/ article/ view/ 1370

O ntañón, T. B. (2016). Circo na escola: por uma educação corporal, estética e artística. (Tesis de doctorado). Faculdade deEducação Física, U niversidadeEstadual de Campinas, Campinas.

O ntañón, T. B., \& Bortoleto, M. A. C. (2014). Todosala pista: El circo en las clases de educación física. Apunts - Educación Física y D eportes, (115), 37-45. Recuperado de https:// www. raco.cat/ index. php/ ApuntsEFE/ article/ view/276018/ 0

O ntañón, T. B., Bortoleto, M. A. C., \& Silva, E. (2013). Educación corporal y estética: lasactividadescircenses como contenido de laEducación Física. Revista Iberoamericana De Educación, (62), 233-43. Recuperado de https: / / rieoei.org/ historico/ documentos/ rie62a13. pdf

Ontañón, T. B., Duprat, R. M., \& Bortoleto, M. A. C. (2012). Educação física e atividades circenses: «0 estado da arte». M ovimento, 18(2), 149-168. DOI https:/ / doi.org/ 10.22456/ 1982-8918.22960

O ntañón, T. B., Lopes, D. C., Santos Rodrigues, G., Cardani, L. T., \& Bortoleto, M. A. C. (2019). Corpo e arte: uma proposta pedagógica na Educação Física a partir da bola de equilíbrio circense. Educación Física Y Ciencia, 2(21). DOI http:/ / dx. doi.org/ 10.24215/23142561e076

Retamal, F. C., Cáceres, K. H., M orales, J. V., \& Muñoz, P.V. (2012). Circo en la escuela: tiempo parala transformación, expansión y significación. Licere, 15(4), 1-15. Recuperado de https:/ / periodicos. ufmg.br/ index. php/ licere/ article/ view/ 695

Santos Rodrigues, G. (2018). Pedagogia das atividades circenses na Educação Física escolar: experiências da arte em escolas brasileiras de Ensino Fundamental (Tesis de maestria). Faculdade de Educação Física, Universidade Estadual de Campinas, Campinas.

-----Santos Rodrigues, G. , Martins, P. P., \& De M arco, A. (2020). Pedagogia das atividades circenses nas atividades extracurriculares: relato de uma experiência de criação e composição coreográfica «A maravilhosa história do Circo». Libro de Actas del Simpósio R eflexões Cênicas Contemporâneas (1-17), (5). Recuperado de https:/ / www. cocen. unicamp.br/ revistadigital/ index.php/ simposiorfc/ article/ view/ 705

Santos Rodrigues, G., Prodócimo, E., \& O ntañón, T. B. (2016). «Circo Coragem»: 0 jogo como estratégia de ensino das atividades circenses. N uances: estudos sobre Educação, 27(1), 147-164. DO I: https:// doi.org/ 10.14572/ nuances. V27i1.3969

Simon, A. (2000). Cycle 2: apprentis equilibristes. Dossier LesArts du Cirque à l'école. Revue EPS1, (97), 19-20.

takamori, F. S., Bortoleto, M. A. C., Liporoni, M. 0., Palmen, M. J. H., \& Cavallotti, T. D. (2010). Abrindo as portas para as atividades circenses na Educação Física escolar: um relato de experiência. Revista Pensar a Prática, 13(1), 1-16. Recuperado de https:/ / www. revistas. ufg. br/ fef/ article/ view/ 6729

Thomas, G. (2011). A typology for the case study in social science following a review of definition, discourse, and structure. Qualitative Inquiry, 17(6), 511-521. DOI https:// doi.org/ 10.1177/ 1077800411409884

Tucunduva, B. (2015). 0 circo na formação inicial em educação física: inovações docentes, potencialidadescircenses. (Tesis de doctorado). Faculdade de Educação Física, Universidade Estadual de Campinas, Campinas.

Tucunduva, B. B., \& Bortoleto, M. A. C. (2019). 0 circo eainovação curricular naformação de professores de Educação Física no brasil. M ovimento, 25, 1-13. DO I https:/ / doi.org/ 10.22456/ 1982-8918.88131 Vendruscolo, C. R. P. (2009). 0 circo na escola. Motriz: Revista de Educação Física, 15(3), 729-737. Recupera do de http:/ / hdl. handle. net/ 11449/ 6757.

\section{(Footnotes)}

${ }^{1}$ Este trabajo fue realizado con el apoyo de la Coordinación de Perfeccionamiento de Personal de N ivel Superior - Brasil (CAPES) - Código de Financiamiento 001.

2 Destacamos que los nombres utilizados para respetar la identidad de las instituciones y de los sujetos de investigación, son ficticios.

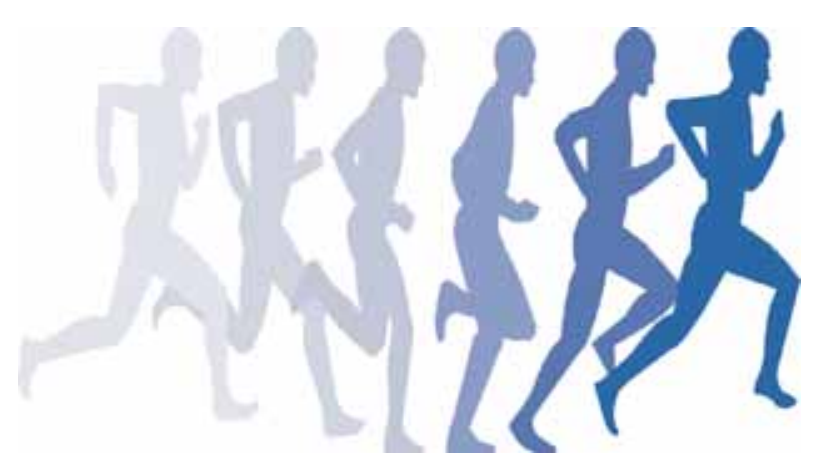

